\title{
Erratum: Fidelity of a sequence of SWAP operations on a spin chain [Phys. Rev. B 102, 035439 (2020)]
}

\author{
Robert E. Throckmorton $\odot$ and S. Das Sarma
}

(Received 19 August 2021; published 3 September 2021)

DOI: 10.1103/PhysRevB.104.119902

We have discovered that some of our numerical results were in error. None of our conclusions in the original paper change as a result of these revised figures. We present here the corrected versions of the results in question.

The corrections that we wish to make to our results are as follows.

In doing our calculations in which we initialize the leftmost spins in the singlet state, $|S\rangle=\frac{1}{\sqrt{2}}(|\uparrow \downarrow\rangle-|\downarrow \uparrow\rangle)$, we in fact performed an additional SWAP gate at the end that interchanges the leftmost two spins. Ideally, this would only result in an overall minus sign on the state, but it still affects the fidelity of the real sequence.

We also found that the right panels of Figs. 3 and 4 of our original paper were incorrect; we present here the corrected figures. The results that we had presented instead initialized the leftmost two spins in a triplet state, $|T\rangle=\frac{1}{\sqrt{2}}(|\uparrow \downarrow\rangle+|\downarrow \uparrow\rangle)$, rather than the intended singlet state.

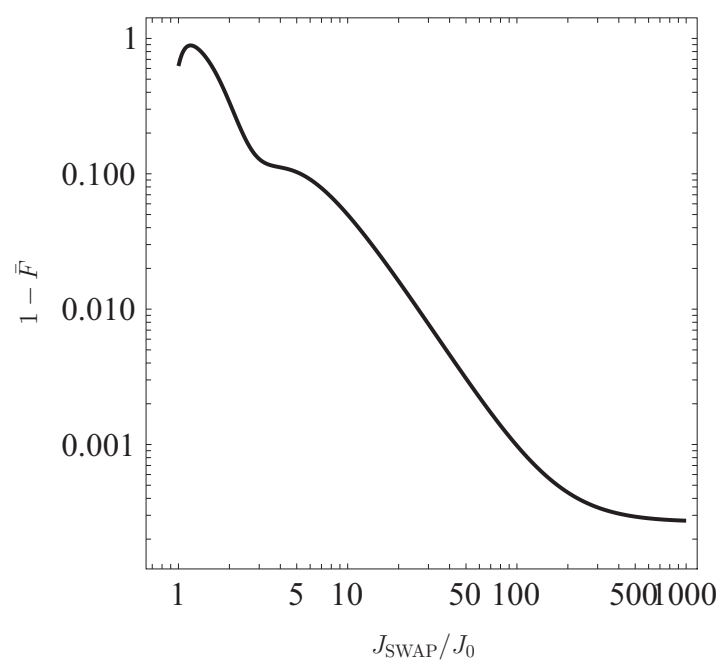

FIG. 3. Corrected version of the right panel of Fig. 3.

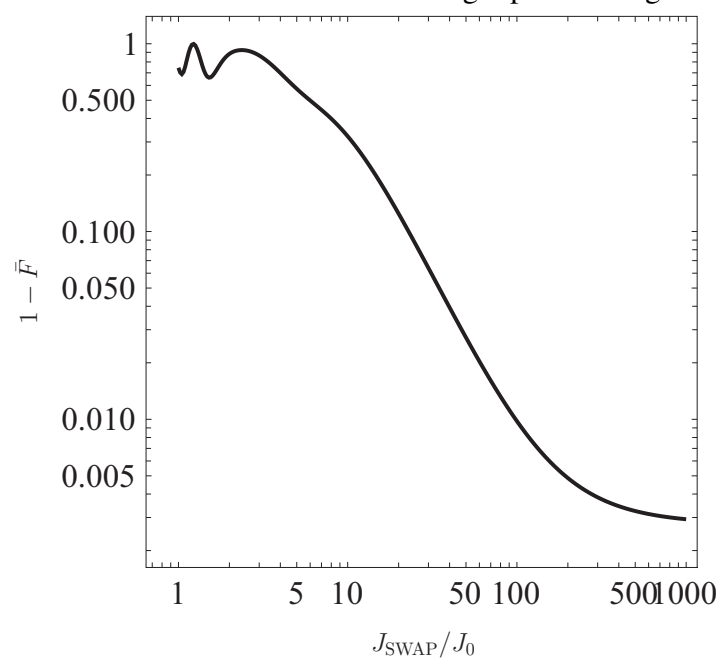

FIG. 4. Corrected version of the right panel of Fig. 4. 


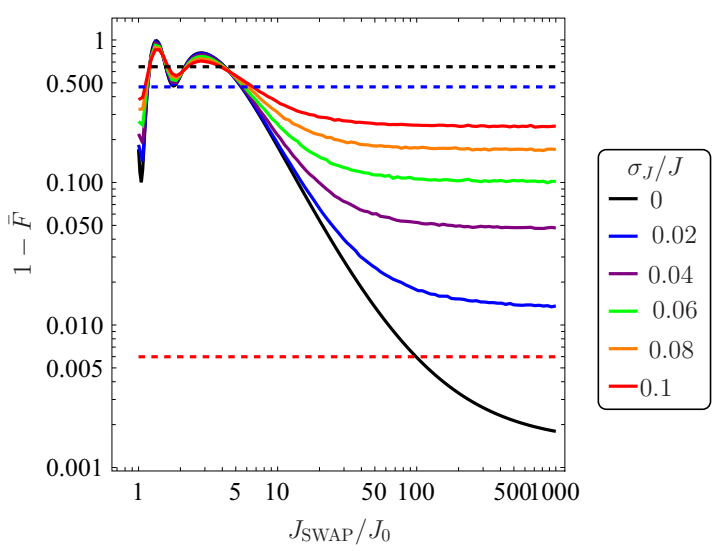

FIG. 5. Corrected version of the right panel of Fig. 5. Note the lower values of $\sigma_{J} / J_{0}$ that we use compared to the original figure.

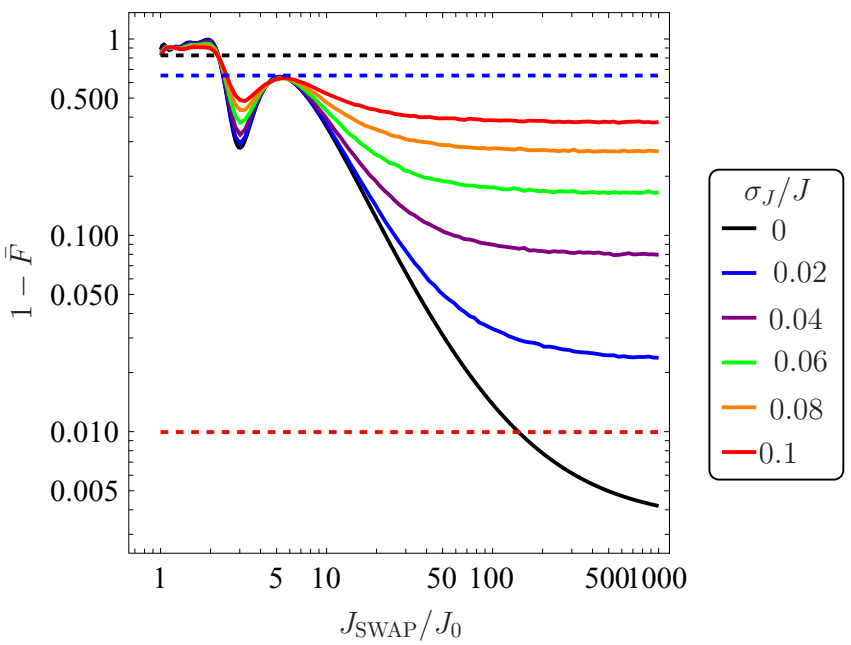

FIG. 6. Corrected version of the right panel of Fig. 6. Note the lower values of $\sigma_{J} / J_{0}$ that we use compared to the original figure.

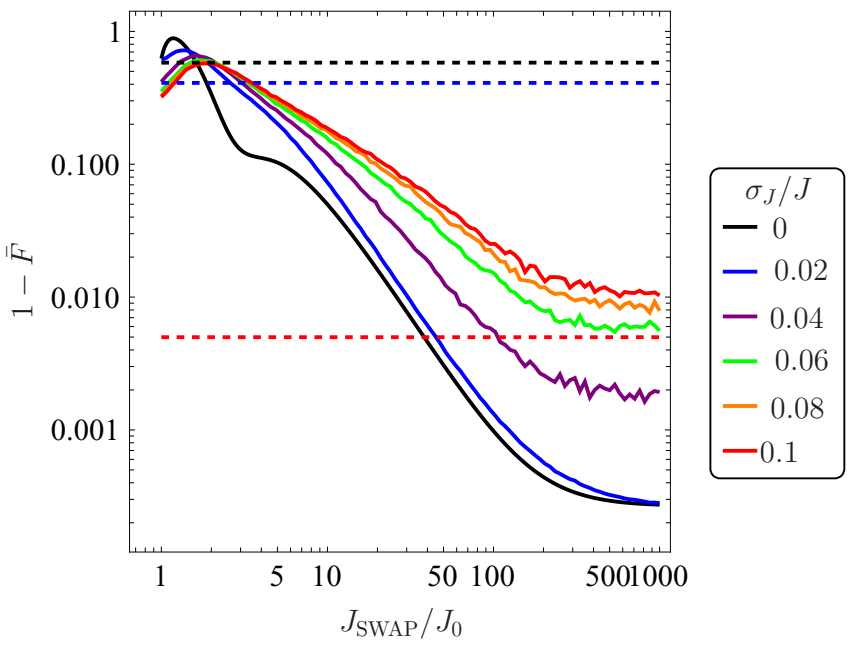

FIG. 7. Corrected version of the right panel of Fig. 7. Note the lower values of $\sigma_{J} / J_{0}$ that we use compared to the original figure. 


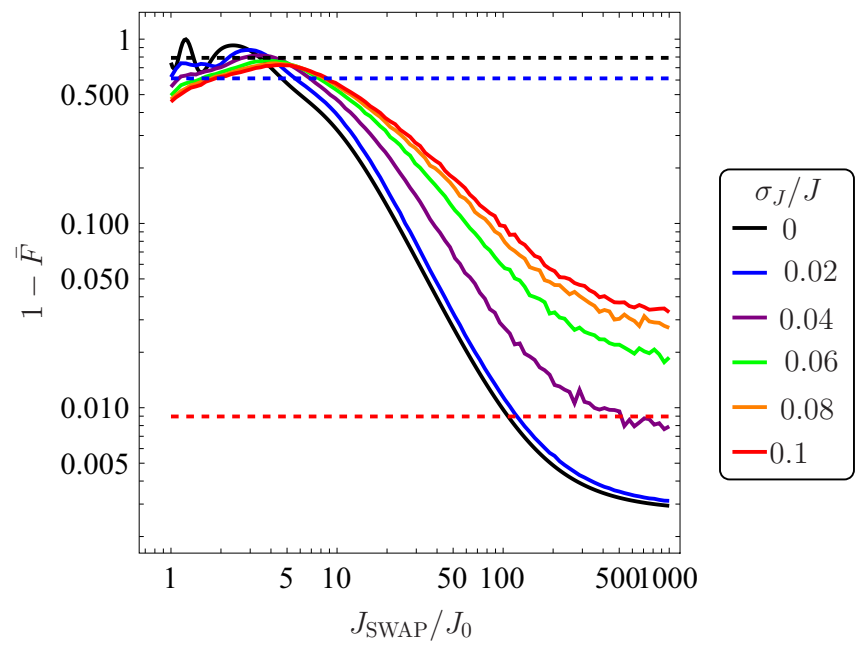

FIG. 8. Corrected version of the right panel of Fig. 8. Note the lower values of $\sigma_{J} / J_{0}$ that we use compared to the original figure.

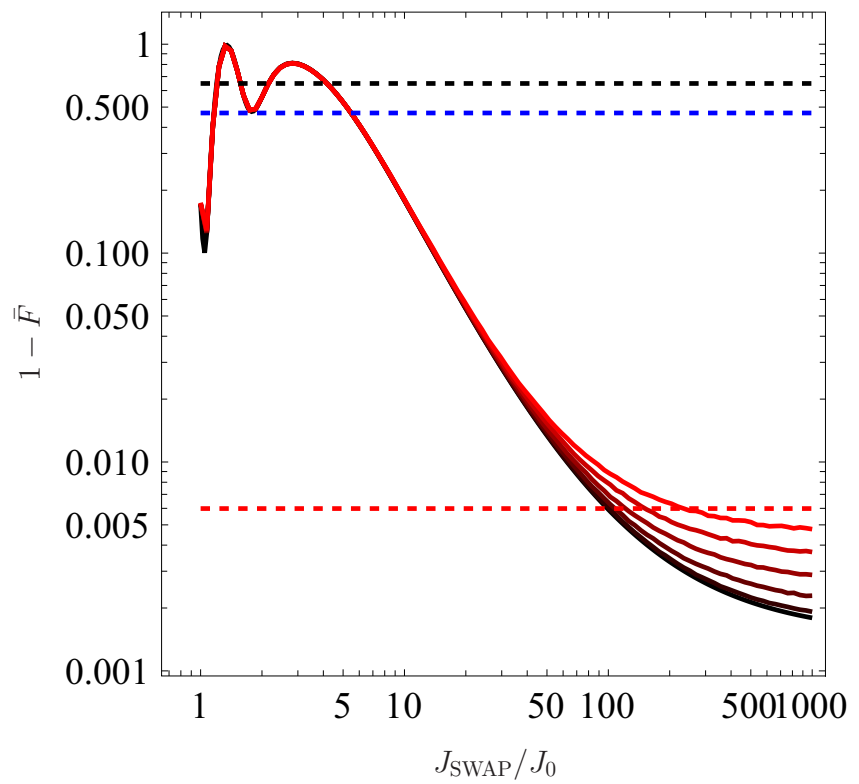

FIG. 9. Corrected version of the right panel of Fig. 9. The curves now cover $\sigma_{J} / J_{0}=0-0.01$ in steps of 0.002 , with the black curve being $\sigma_{J}=0$ and the red curve being $\sigma_{J} / J_{0}=0.01$.

Finally, our figures showing our results with noise, Figs. 5-9 of our original paper, were in error; we present here the corrected figures.

We thank Nathan Lars Foulk for pointing out the errors corrected here. This work was supported by the Laboratory for Physical Sciences. 